\title{
Modality Switch Effects Emerge Early and Increase throughout Conceptual Processing: Evidence from ERPs
}

\author{
Pablo Bernabeu (pcbernabeu@gmail.com) \\ Centre for Language Studies, Radboud University \\ Erasmusplein 1, 6525 HT - Nijmegen, Netherlands \\ Roel M. Willems (r.willems@let.ru.nl) \\ Neurobiology of Language dept., Max Planck Institute for Psycholinguistics \\ Wundtlaan 1, 6525 XD - Nijmegen, Netherlands
}

\author{
Max M. Louwerse (m.m.louwerse@tilburguniversity.edu) \\ Tilburg center for Cognition and Communication, Tilburg University \\ Warandelaan 2, 5037 AB - Tilburg, Netherlands
}

\begin{abstract}
We tested whether conceptual processing is modality-specific by tracking the time course of the Conceptual Modality Switch effect. Forty-six participants verified the relation between property words and concept words. The conceptual modality of consecutive trials was manipulated in order to produce an Auditory-to-visual switch condition, a Haptic-tovisual switch condition, and a Visual-to-visual, no-switch condition. Event-Related Potentials (ERPs) were time-locked to the onset of the first word (property) in the target trials so as to measure the effect online and to avoid a within-trial confound. A switch effect was found, characterized by more negative ERP amplitudes for modality switches than noswitches. It proved significant in four typical time windows from 160 to 750 milliseconds post word onset, with greater strength in posterior brain regions, and after 350 milliseconds. These results suggest that conceptual processing may be modality-specific in certain tasks, but also that the early stage of processing is relatively amodal.
\end{abstract}

Keywords: conceptual processing; time; modality switch; perceptual simulation; amodal; event-related potentials; ERP

\section{Introduction}

Research in the cognitive sciences has extensively investigated whether conceptual processing is modalityspecific (Barsalou, 2016). In a commonly used paradigm known as the Conceptual Modality Switch (CMS), participants perform a property verification task in which they decide whether certain property words can reasonably describe certain concept words. For instance, Pecher, Zeelenberg, and Barsalou (2003) presented sentences such as Blenders can be loud. Covertly, the conceptual modality of consecutive trials was manipulated in order to produce specific switches. A sentence like Blenders can be loud, which is mainly related to the auditory modality, could either be followed by a sentence within the same modalitye.g., Leaves can be rustling-, or by a sentence in a different modality-e.g., Cranberries can be tart (gustatory). Pecher et al. found that when the modalities of consecutive trials did not match, participants took longer to respond. Such an effect suggested that perceptual features of concepts (operationalized in the modality shifts) are accessed during conceptual processing. More recently, however, the CMS effect was reanalysed using a nonperceptual alternative, language statistics (i.e., how words co-occur in a language). Louwerse and Connell (2011) found that language statistics were able to approximately predict what modality a concept and property pair belonged to. Specifically, they could predict a visual/haptic modality, an olfactory/gustatory modality, and an auditory modality, but they could not predict the subtler differences between visual and haptic, and between olfactory and gustatory, which seemed to be reserved for perceptual simulations. Moreover, when a language statistics explanation and a perceptual explanation were compared against one another, faster response times (RTs) were best explained by language statistics, whereas slower RTs were best explained by perceptual simulations (for similar findings with switches in emotion, see Tillman, Hutchinson, Jordan, \& Louwerse, 2013). Louwerse and Hutchinson (2012) further replicated these findings in an Electroencephalography (EEG) experiment in which they showed that those cortical regions commonly associated with language processing are relatively more active in the beginning of processing, whereas those regions commonly associated with perceptual processing are relatively more active later on. These studies demonstrated that the time course of processing is important in the study of language statistics and perceptual simulation.

\section{Time Course of Effects in Word Processing}

The time course of word processing may be relevant for an effect such as the CMS. Hauk (2016) zooms into the one second during which a word is processed, proposing the following timeline. A reader or listener starts to identify a word and to access part of its meaning within around 150 milliseconds (ms) from word onset. Building on that information, working memory processes emerge at around $170 \mathrm{~ms}$ post word onset, followed by response-related processes at around $250 \mathrm{~ms}$. Mental imagery and episodic memory are the last-emerging processes, both around 400 ms post word onset. Once started, each of these processes extends further, gradually overlapping with each other. This 
timeline suggests two important things about the earlier and the later stages of word processing. First, having an early emergence-e.g., at $250 \mathrm{~ms}$ - does not make an effect lexicosemantic per se because the meaning encoded could have gone through working memory before activating the actual system of interest, e.g., sensorimotor (Mahon \& Caramazza, 2008). Second, it suggests that effects emerging later face that same challenge and, in addition, the potential influence of response-related and forthcoming processes.

If even an effect measured online with high temporal resolution (EEG or Magnetoencephalography) may be subject to alternative causation, effects measured with lower temporal resolution (functional Magnetic Resonance Imaging) or off-line (RT) are arguably more challenged by this. With regard to the current topic specifically, sensory and motor effects may possibly be epiphenomenal to the representation of concepts online, independently of what is suggested by the measurements off-line or online lagged.

There is a technique especially apt for testing the causality of cognitive systems, namely, Transcranial Magnetic Stimulation (TMS). Willems et al. (2011) found that comprehension of hand-related verbs was improved as a consequence of stimulating the hand area of premotor cortex. It was particularly improved when the TMS was applied in the hemisphere controlling the dominant hand. More recently, Vukovic et al. found an impairment in the processing of action-related words, along with an improvement in the processing of abstract words, after TMS was applied over motor cortex, $200 \mathrm{~ms}$ after word onset. The latter finding suggests that the contribution of modalityspecific systems (in this case, motor ones) can emerge relatively early (see also Amsel, Urbach, \& Kutas, 2014; Van Dam, Brazil, Bekkering, \& Rueschemeyer, 2014).

In our view, two interlocked questions stand out in the current topic area. The causality question asks whether modality-specific effects reflect a functionally relevant simulation process or arise only after basic conceptual processing has been attained. The compatibility question asks whether different processing systems, amodal and modal, may compatibly operate in conceptual processing.

\section{Experiment}

We addressed the causality and the compatibility questions by revisiting the CMS paradigm (see most recent previous study in Scerrati, Lugli, Nicoletti, \& Borghi, 2016).

\section{Tracking the Time Course of the CMS}

We measured the CMS online by time-locking EventRelated brain Potentials (ERPs) to the onset of the first word in the target trials. We wanted to establish where exactly the effect-indexing access to perceptual informationemerged, how far it extended, and the relative strength over the time course. These measures would allow us to relatively assess how strongly the CMS may be influenced by response-related and other extra-semantic processes (see Hauk, 2016). Concerning the compatibility question, previous research would predict an increase in the CMS effect over time because earlier processing is relatively amodal (Louwerse \& Hutchinson, 2012).

The three previous ERP studies on the CMS time-locked the measurement to property words placed last in the target trials (Hald, Marshall, Janssen, \& Garnham, 2011; Collins, Pecher, Zeelenberg, \& Coulson, 2011; Hald, Hocking, Vernon, Marshall, \& Garnham, 2013). A potential problem of those measurements is a lack of certainty on the emergence of the effect, because a switch might reasonably emerge already at the first content word in the target trial. Therefore, in our design we placed the property word first in the target trial, and time-locked ERPs to its onset. This had an important advantage, as it helped avoid a confound caused by the relation between the property and the concept in each target trial (see Hald et al., 2013). The possibility of those two confounds - the lagged measurement and the within-trial relationship — could explain why the CMS effect has sometimes failed to appear in RTs (Hald et al., 2011; 2013; Collins et al., 2011; Scerrati et al., 2016).

We did not have clear hypotheses on what we would find as the time course of the CMS because we were the first to time-lock ERPs to the first word. Nonetheless, the effects found in the three ERP studies cited above were generally characterized as $\mathrm{N} 400$ - linked to semantic violation-, with more negative amplitudes for modality switches than noswitches. The earliest emerging effect appeared in Hald et al. (2011), in a time window from 270 to $370 \mathrm{~ms}$.

\section{Different Switches and Processing Speeds}

In order to further explore the compatibility question, we drew on Louwerse and Connell (2011). As reviewed in the Introduction, they found that quick processing was able to pick up most switch types but missed the subtler ones, for instance, between haptic and visual. By contrast, slow processing had the advantage of picking up even those subtler switches. Here we brought these findings to a group design. We distinguished a Quick group of participants and a Slow group of participants based on their average RT. Maintaining the CMS as a within-subjects factor, we predicted that the larger modality switches (e.g., auditory to visual) would be picked up equally by both groups, whereas the subtler switches (e.g., haptic to visual) would be picked up only — or more clearly - by the Slow group.

\section{Method}

Accuracy Pretest The task was validated in a behavioural pretest ( $N=19$; Radboud U., Tilburg U.) revealing that all participants but one had an average response accuracy over $50 \%$, and the overall average was $63 \%(S D=48 \mathrm{pp}$.$) .$

Participants Forty-nine participants - native speakers of Dutch with no relevant disorders-were recruited at the Max Planck Institute for Psycholinguistics. They were paid a small fee after participating. Participants were randomly assigned to one of three experimental groups: a Quick response group $(n=22)$, a Self-paced response group $(n=$ 21 ), and a Null group who got the same experimental design 
as the Self-paced group but no instructions on response speed $(n=5)$ (see Figure 1). One participant had to prematurely leave the experiment. Another participant had to be removed from the data due to too noisy ERPs (7 retained trials out of 108). Under visual inspection, all other participants' waveforms-preprocessed and averaged per CMS condition - approximately presented the typical peaks of word reading. Last, one participant, the only one with an accuracy below 50\%-i.e., $37 \%$-, was also removed from further analyses. Forty-six participants remained. Because the Groups presented rather close, significantly equal RTs, we pooled them together and re-split them in two groups on the basis of each participant's average RT. The effects CMS and CMS by Group were equally significant with the old and new groups in both ERPs and RTs. New groups were: Quick $(n=23$; mean RT $=568.40 \mathrm{~ms}, S D=104.83$; age 1931 , mean $=23.3 ; 19$ females $)$, and Slow $(n=23$; mean RT $=$ $937.21 \mathrm{~ms}, S D=265.56$; age $18-25$, mean $=22.2 ; 18$ females). The different tests-stimulus norming, pretest, and main experiment—-did not share any participants.

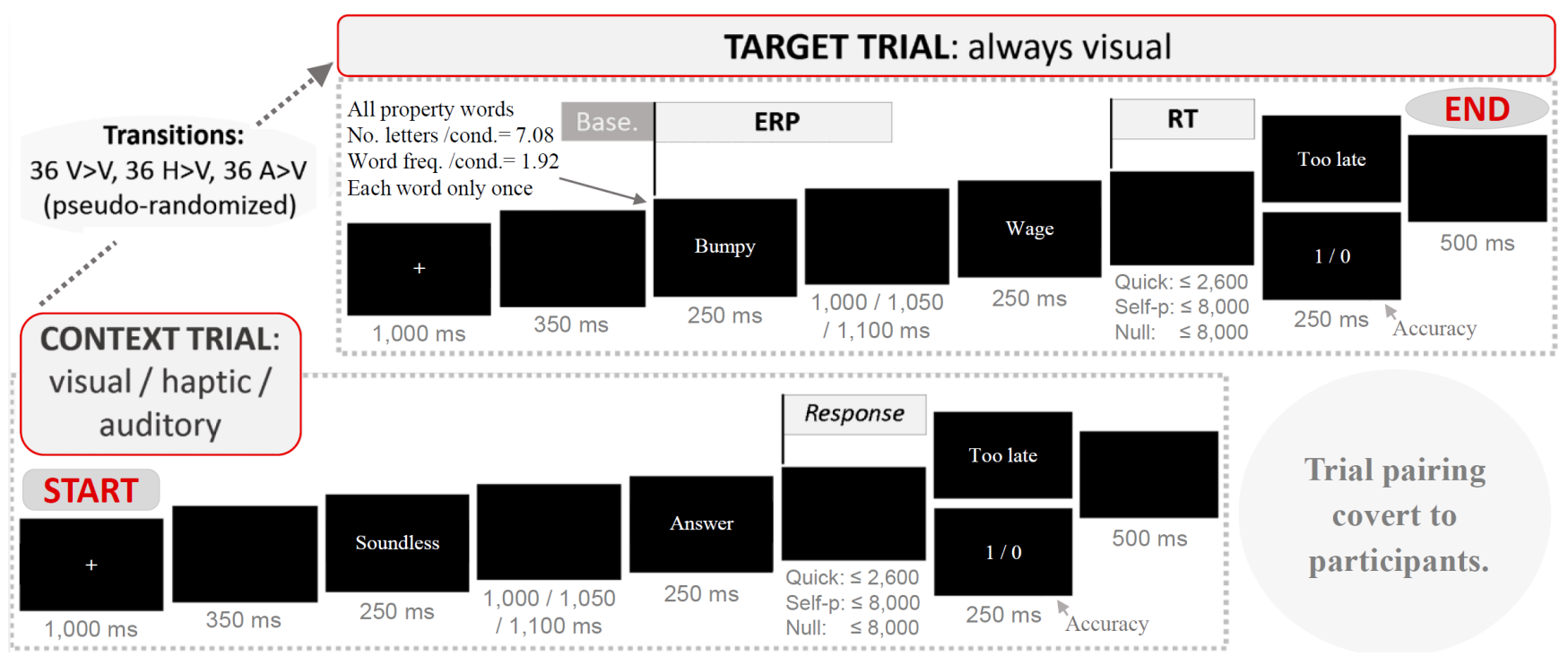

Figure 1: Schema illustrating materials, design, and procedure. Note that Groups were pooled and re-split (see Participants).

Materials and Design As in previous CMS studies, the stimuli consisted of pairs of property and concept words, but we had a small novelty in this combination. Whereas previous studies presented the concept and the property in declarative sentences (Pecher et al., 2003; Louwerse \& Connell, 2011; Hald et al., 2011), or with the concept followed by the property alone (Collins et al., 2011), the current experiment presented the property followed by the concept alone, e.g., Soundless Answer. In this design, as in most, the property word took the most relevant position for the measurement, because properties are generally more modality-specific than concepts (Lynott \& Connell, 2013).

The properties and concepts, all in Dutch, were partly based on Lynott and Connell's (2009, 2013) norms. We normed our items similarly too, by asking forty-two respondents to rate 0 to 5 the extent to which they experienced each property or concept with the senses of hearing, touch, and vision. Then we computed the dominant modality of each word (Bernabeu, Louwerse, \& Willems, 2017). Next, we created 216 trials by joining properties and concepts within the same modalities. ${ }^{1}$ Half of the trials contained a fairly related property and concept, while the other half presented rather unrelated pairs. These

${ }^{1}$ Olfactory and gustatory words were not used because there were too few in our candidate stimuli, and were not required. relationships served to engage participants in a semantic task, yet conveniently did not affect the ERP measurement because ERPs were measured before the concept word was presented in each trial (Figure 1). In spite of this, we wish to acknowledge that some trials came out rather unnaturalLukewarm Volume - or fuzzy-Solid Ideal-because they were created out of a fixed set of modality norms (Bernabeu et al., 2017). In order to alleviate that problem, the instructions of the experiment stated that the accuracy feedback following every response was based on the answers of previous participants (in reality, it wasn't). Furthermore, the stimuli and the task were validated by the accuracy rates in the pretest and in the main experiment.

For the critical CMS manipulation, trials were covertly paired as context and target trials. This was done pseudorandomly within participants and CMS conditions by using the software Presentation ${ }^{\mathrm{TM}}$. Three conditions were created-Auditory-to-visual, Haptic-to-visual, and Visualto-visual-, each with 36 context trials and 36 target trials. One auditory-to-visual switch for one participant was: Soundless Answer | Bumpy Wage (bold added to ERP-target word). For another participant, the latter target trial was instead preceded by the context trial Loud Welcome. The pseudo-randomization ensured that ERP-target words (properties) were matched across CMS conditions on the 
essential criteria-word frequency and length (letters), and semantic class. Also, ERP-target words occurred only once.

Procedure The entire experiment was in Dutch. By means of written instructions, participants were asked to respond in each trial whether the first word, a property, could be used to describe the second word, a concept. Two buttons were used to respond. An example was provided based on the property 'grey' and the concept 'snow.' Snow is often white, but it can also be grey. By contrast, a property that would not match is 'pink.' Then, the instructions diverged for the different groups of participants: while the Quick group was asked to respond as quickly as possible in every trial, the Self-paced group was asked to respond self-paced, and the null group was altogether unconstrained (see design constraints for each group in Figure 1). Further, the instructions stated that feedback would be provided for each response, and that this was based on all preceding answers (although it was not), and therefore participants need not worry too much about mistakes. Last, they were asked to move or blink as little as possible, and do so only while the cross was on the screen. Twelve practice trials ensued, after which participants could ask questions. The experiment ran on Presentation ${ }^{\mathrm{TM}}$. The experiment proper lasted about 20 minutes, with a break in the middle. Taking into account EEG procedures, it lasted about 1 hour and 45 minutes.

ERP Recording and Preprocessing The EEG signal was recorded with BrainVision Recorder $1^{\mathrm{TM}}$, in differential mode, utilizing 65 active $\mathrm{Ag} / \mathrm{AgCI}$ electrodes. The ground electrode was positioned just above the nose, at the glabella. Three other electrodes were used to register eye movements, two placed at the outer canthi of each eye, and one placed below the left eye. The remaining 59 electrodes were mounted in a custom, equidistant ActiCap cap. Impedance was kept below $10 \mathrm{k} \Omega$ by applying electrolyte gel at the tip of each electrode. The signal was amplified through BrainAmp DC amplifiers with a bandpass filter of $0.016-$ $100 \mathrm{~Hz}$, and an online sampling frequency of $500 \mathrm{~Hz}$ (i.e., every $2 \mathrm{~ms}$ ). Afterwards, the signal was preprocessed in BrainVision Analyzer $2^{\mathrm{TM}}$, with the following steps: CMS condition segmentation, automatic ocular correction, $200 \mathrm{~ms}$ baseline correction, artefact rejection via semi-automatic segment selection. ${ }^{2}$ The proportion of segments (trials) retained from the 46 final participants was: $77.4 \%$ in the Visual-to-visual condition, $78.0 \%$ in the Haptic-to-visual condition, and $78.6 \%$ in the Auditory-to-visual condition.

\footnotetext{
${ }^{2}$ Segment selection (partly based on the Brain Vision Analyzer tutorials at http://www.erpinfo.org/the-erp-bootcamp.html). The critical period spanned from $300 \mathrm{~ms}$ before target onset to 800 $\mathrm{ms}$ after target onset (the period before onset is $100 \mathrm{~ms}$ longer than the general baseline of the ERPs because that improved the selection of segments). Gradient: $75 \mu \mathrm{V} / \mathrm{ms}$. Threshold for difference between maximum and minimum voltage in segment: $\pm 150 \mu \mathrm{V}$ (this was increased or decreased by up to $40 \mu \mathrm{V}$ in a minority of cases where the automatic selection yielded too noisy waveforms), interval length $200 \mathrm{~ms}$. Amplitude: $-100 \mu \mathrm{V}$, $+100 \mu \mathrm{V}$. Low activity: $0.5 \mu \mathrm{V}$, interval length $50 \mathrm{~ms}$.
}

ERP Analysis The ERPs, averaged per CMS condition, were downsampled to $125 \mathrm{~Hz}$ due to computational demands. Electrodes were divided into an anterior and a posterior area (also done in Hald et al., 2011). Albeit a superficial division, we found it sufficient for the research question. Time windows were selected as in Hald et al., except for the last window, which was extended up to 750 $\mathrm{ms}$ post word onset, instead of $700 \mathrm{~ms}$, because the characteristic component of that latency tends to extend until then, as we confirmed by visual inspection of these results. Window 1 was meant to capture N1-P2 components, Window 2 the pre-N400, Window 3 the N400, and Window 4 the LPC/P600. Analyses were performed in R.

\section{Results}

All final participants responded correctly in over half of the trials. The average accuracy was $63 \%(S D=48$ pp. $)$, nearly identical in each participant Group and CMS condition. ${ }^{3}$

ERPs The ERP results revealed a CMS effect from Time Window 1 on, larger after $350 \mathrm{~ms}$. It appeared with both switch conditions, and was characterized by a more negative amplitude for the switch conditions compared to the noswitch condition. In certain parts over the time course, the effect appeared in both anterior and posterior areas, and in both participant groups, but it was generally stronger in the posterior area and in the Slow group (Figure 2).

ERPs were analyzed per time window by means of Linear Mixed Effects models (lme4 R package). Random intercepts and slopes, and fixed effects, were tested with the critical factors and interactions, as well as with potential confounds, e.g., handedness, sex, age. Each inclusion was tested in a stepwise fashion based on the significance of the Likelihood Ratio. The final models presented good fits, with $R^{2}$ ranging from .748 (Window 4) to .862 (Window 2). Table 1 sums up the results. First, the CMS effect in Time Window 1 was confirmed significant (see detailed waveforms in Figure 3). Such an early emergence is unprecedented in the CMS literature, and it may have been enabled by the time-locking of ERPs to the first word in target trials. In this time window, the only process not lexicosemantic is possibly working memory (Hauk, 2016), and therefore this early emergence lends support to the possibility that the CMS had a lexicosemantic basis (but see Mahon \& Caramazza, 2008).

Whereas in Time Window 1 (160-216 ms), the CMS effect was circumscribed to an interaction with Brain Area (anterior/posterior), by Window 2 (270-370 ms) a main effect of CMS emerged. Finally, in Window 3 (350-550 $\mathrm{ms})$ and Window 4 (500-750 ms), the only critical effect was CMS. Window 3 presented the largest main effect of CMS. Planned ANOVA contrasts into CMS conditions, corrected for multiple comparisons, revealed that the noswitch condition differed significantly from the switches.

\footnotetext{
${ }^{3}$ Quick group: Auditory-to-visual: $62 \%$ ( $S D=48$ pp.). Haptic-tovisual: $61 \%$ ( $S D=49$ pp.). Visual-to-visual: $63 \%$ ( $S D=48$ pp.). Slow group: Auditory-to-visual: $64 \%$ ( $S D=48$ pp.). Haptic-tovisual: $64 \%$ ( $S D=48$ pp.). Visual-to-visual: $64 \%$ ( $S D=48$ pp.).
} 

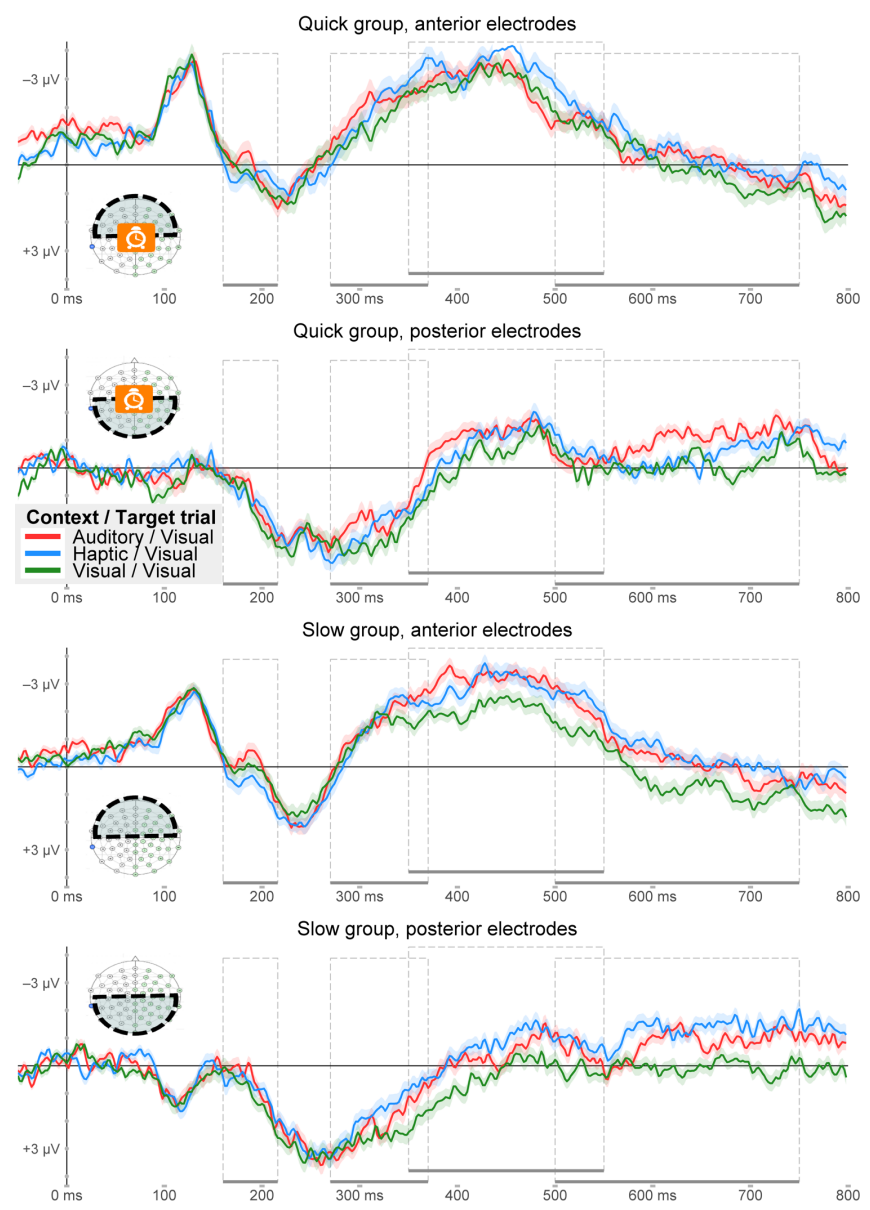

Figure 2: Data per Group and Area, with 95\% Confidence Intervals every $2 \mathrm{~ms}$, and time windows. Negative up.

Table 1: Effect of CMS and its interaction with Ant./Pos. brain Area and with Group. ${ }^{* * *} p<.001 ;{ }^{* *} p<.01 ;{ }^{*} p<.05$.

\begin{tabular}{|c|c|c|}
\hline Time window & Factors & Effect: $\chi^{2}$ \\
\hline \multirow{3}{*}{ 1) $160-216 \mathrm{~ms}$} & $\mathrm{CMS}$ & 1.40 \\
\hline & CMS x Ant/Pos Area & $48.59^{* * *}$ \\
\hline & CMS x Ant/Pos Area x Group & $23.63^{* *}$ \\
\hline \multirow{3}{*}{ 2) $270-370 \mathrm{~ms}$} & CMS & $6.40^{*}$ \\
\hline & CMS x Ant/Pos Area & $10.89^{* *}$ \\
\hline & CMS x Ant/Pos Area x Group & $4.13^{* * *}$ \\
\hline 3) $350-550 \mathrm{~ms}$ & CMS & $9.47^{* *}$ \\
\hline 4) $500-750 \mathrm{~ms}$ & CMS & $7.58^{*}$ \\
\hline
\end{tabular}

By contrast, the switch conditions hardly differed from each other-statistically equal in some sections of the data-, fitting the CMS effect. The fit of these follow-up ANOVAs was high in Windows 1 to 3, and medium in Window 4.

The interaction of Group and CMS was only significant in Time Windows 1 and 2. Yet, the waveforms in Windows 2, 3 , and 4 presented a pattern which precisely converged with our hypothesis based on Louwerse and Connell. Whereas

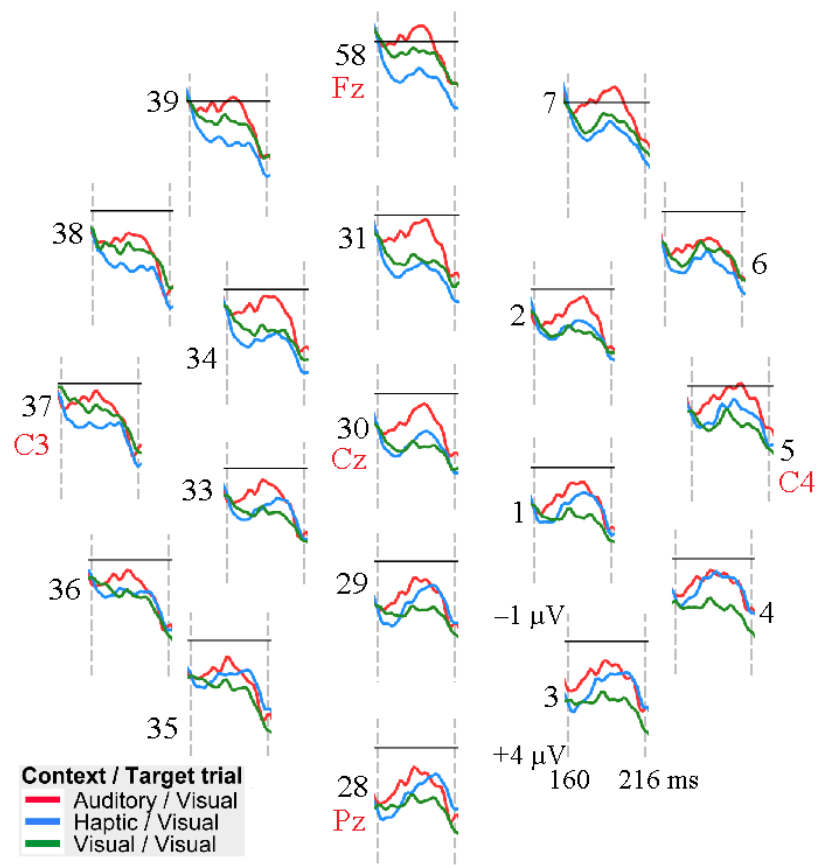

Figure 3: Subset of electrodes from the Slow group at Time Window 1 (the Quick group presented a slightly smaller but also significant effect). $\mathrm{Y}$-axis ranges from $-1 \mu \mathrm{V}$ to $+4 \mu \mathrm{V}$. Red labels signal the equivalents in the 10-20 montage.

the Slow group picked up the switches across all modalities similarly, the Quick group presented a larger switching cost for the Auditory-to-visual switch relative to the Haptic-tovisual switch. This interaction would support a compatibility of distributional and perceptual processing.

RTs This design was tailored to measure ERPs. RTs were not reliable enough regarding the CMS because the last word in the target trials - critical for RTs-had not been matched across conditions on the essential criteria (see Materials section above). Nonetheless, we analysed RTs, statistically controlling for the confounds. No effects involving CMS were found, all $p \mathrm{~s}>.05$ (model $\left.R^{2}=.552\right)$.

\section{Discussion}

The Conceptual Modality Switch effect is a largely replicated demonstration of the relevance of modalityspecific information for conceptual processing. We tracked this effect online in order to ascertain at what stages perceptual information is processed, and to what degree (see Mahon \& Caramazza, 2008; Hauk, 2016). Time-locking ERPs to the onset of the first word in the target trials brought the added advantages of cancelling confounds within the target trial and measuring the effect at the onset, un-lagged. On the other hand, this design had the disadvantage of some unnatural stimuli. In spite of these novelties, though, our broad randomization of trials and the results found suggest that this experiment preserved the essence of the CMS paradigm. We found the CMS effect emerging at the start of lexicosemantic and working 
memory processing, then increasing through the rest of word processing. The virtually immediate effect upon word recognition offers further support for the suggestion that sensory brain regions have a functional role in conceptual processing, at least in a fairly demanding semantic analysis as in the current task (see Louwerse \& Hutchinson, 2012). Solving the causality question, nonetheless, may require in the future more fundamental research on word processing, in addition to TMS-based work, in order to qualify the degree of semantic and post-semantic processing in an effect (see Hauk, 2016). The increase in the CMS effect over the time course converges with previous findings in suggesting that distributional processing-language statistics - may play a greater role earlier on (Louwerse \& Connell, 2011; Louwerse \& Hutchinson, 2012). This earlylate distribution fits with Hauk's (2016) word processing timeline, where the early stage has a greater relative proportion of lexicosemantic processing, which would presumably support language statistics. Increasing evidence on the compatibility of amodal and modal/embodied processing invites further research. Concerning the CMS specifically, we still need to establish whether this effect can best be explained by language statistics or by perceptual simulations. The current work at least demonstrates that it emerges early and increases throughout word processing.

\section{Supplementary Material, Acknowledgments}

All data, including the stimuli, set-up, raw files, and results in detail, are available through https://osf.io/97unm/

We received funding from the Neurobiology of Language department at Max Planck Institute for Psycholinguistics, Experimental Psychology Society, Cognitive Science Society, and Tilburg University. We received valuable advice from Brain Products, Gwilym Lockwood, Johan Weustink, Monique Flecken, Ronald Fischer, Sean Roberts, and the peer-reviewers. Also, thanks to our participants.

\section{References}

Amsel, B. D., Urbach, T. P., \& Kutas, M. (2014). Empirically grounding grounded cognition: the case of color. Neuroimage, 99, 149-157.

Barsalou, L. W. (2016). On staying grounded and avoiding quixotic dead ends. Psychonomic Bulletin \& Review, 23.

Bernabeu, P., Louwerse, M. M., \& Willems, R. M. (2017, April 11). Modality exclusivity norms for 747 properties and concepts in Dutch: a replication of English http://doi.org/10.17605/OSF.IO/BRKJW

Collins, J., Pecher, D., Zeelenberg, R., \& Coulson, S. (2011). Modality switching in a property verification task: an ERP study of what happens when candles flicker after high heels click. Frontiers in Psychology, 2.

Hald, L. A., Hocking, I., Vernon, D., Marshall, J.-A., \& Garnham, A. (2013). Exploring modality switching effects in negated sentences: further evidence for grounded representations. Frontiers in Psychology, 4, 93.

Hald, L. A., Marshall, J.-A., Janssen, D. P., \& Garnham, A. (2011). Switching modalities in a sentence verification task: ERP evidence for embodied language processing. Frontiers in Psychology, 2.

Hauk, O. (2016). Only time will tell-Why temporal information is essential for our neuroscientific understanding of semantics. Psychonomic Bulletin \& Review, 23, 4, 1072-1079.

Louwerse, M., \& Connell, L. (2011). A taste of words: Linguistic context and perceptual simulation predict the modality of words. Cognitive Science, 35, 2, 381-98.

Louwerse, M., \& Hutchinson, S. (2012). Neurological evidence linguistic processes precede perceptual simulation in conceptual processing. Frontiers in Psychology, 3, 385. doi:10.3389/ fpsyg.2012.00385

Lynott, D., \& Connell, L. (2009). Modality exclusivity norms for 423 object properties. Behavior Research Methods, 41, 2, 558-564.

Lynott, D., \& Connell, L. (2013). Modality exclusivity norms for 400 nouns: The relationship between perceptual experience and surface word form. Behavior Research Methods, 45, 516-526.

Mahon, B. Z., \& Caramazza, A. (2008). A critical look at the Embodied Cognition Hypothesis and a new proposal for grounding conceptual content. Journal of Physiology Paris, 102, 59-70.

Pecher, D., Zeelenberg, R., \& Barsalou, L. W. (2003). Verifying different-modality properties for concepts produces switching costs. Psychological Science, 14, 2, 119-24.

Scerrati, E., Baroni, G., Borghi, A. M., Galatolo, R., Lugli, L., \& Nicoletti, R. (2015). The modality-switch effect: visually and aurally presented prime sentences activate our senses. Frontiers in Psychology, 6, 1668.

Scerrati, E., Lugli, L., Nicoletti, R., \& Borghi, A. M. (2016). The Multilevel Modality-Switch Effect: What Happens When We See the Bees Buzzing and Hear the Diamonds Glistening. Psychonomic Bulletin \& Review, doi:10.3758/s13423-016-1150-2.

Tillman, R., Hutchinson, S., Jordan, S., \& Louwerse, M. M. (2013). Verifying properties from different emotions produces switching costs: Evidence for coarse-grained language statistics and fine-grained perceptual simulation. Proceedings of the 35th Annual Conference of the Cognitive Science Society (pp. 3551-3556). Austin, TX: Cognitive Science Society.

Van Dam, W. O., Brazil, I. A., Bekkering, H., \& Rueschemeyer, S.-A. (2014). Flexibility in embodied language processing: context effects in lexical access. Topics in Cognitive Science, 6, 407-424.

Vukovic, V., Feurra, M., Shpektor, A., Myachykov, A., \& Shtyrov, Y. (2017). Primary motor cortex functionally contributes to language comprehension: An online rTMS study. Neuropsychologia, 96, 222-229.

Willems, R. M., Labruna, L., D., Esposito, M., Ivry, R., \& Casasanto, D. (2011). A functional role for the motor system in language understanding: Evidence from ThetaBurst Transcranial Magnetic Stimulation. Psychological Science, 22, 849-854. 